FUS-associated amyotrophic lateral sclerosis. Acta Neuropathol 2017; 133: 887906.

Neumann M, Sampathu DM, Kwong LK, Truax AC, Micsenyi MC, Chou TT, et al. Ubiquitinated TDP-43 in frontotemporal lobar degeneration and amyotrophic lateral sclerosis. Science 2006 314: 130-3.
Scekic-Zahirovic J, Sendscheid O, El Oussini H, Jambeau M, Sun Y, Mersmann S, et al. Toxic gain of function from mutant FUS protein is crucial to trigger cell autonomous motor neuron loss. EMBO J 2016; 35: 1077-97.

Tyzack GE, Luisier R, Taha DM, Neeves J, Modic M, Mitchell JS, et al. Widespread FUS mislocalization is a molecular hallmark of amyotrophic lateral sclerosis. Brain 2019; 142: 2572-80.

Vance C, Rogelj B, Hortobágyi T, De Vos KJ, Nishimura AL, Sreedharan J, et al. Mutations in FUS, an RNA processing protein, cause familial amyotrophic lateral sclerosis type 6. Science 2009; 323: 1208 11.

\title{
Inclusion body myositis: accumulation of evidence for its autoimmune origin
}

This scientific commentary refers to 'Highly differentiated cytotoxic $T$ cells in inclusion body myositis', by Greenberg et al. (doi:10.1093/brain/ awz207).

Inclusion body myositis (IBM) is a slowly progressive idiopathic inflammatory myopathy, the most frequent among myopathies (inherited or acquired) in patients over 50 years of age. It is particularly resistant to conventional immunosuppressive drugs, including corticosteroids. IBM was first described in the 1970s, but the first diagnostic criteria based largely on pathology were published only in 1995. Machine learning approaches were subsequently used to construct diagnostic criteria with $90 \%$ sensitivity and $96 \%$ specificity (Lloyd et al., 2014). These criteria are the presence of finger flexor or quadriceps weakness, endomysial inflammation, and $\mathrm{T}$ cell invasion of non-necrotic muscle fibres or rimmed vacuoles (Lloyd et al., 2014). Now well-accepted, these criteria emphasize muscle involvement, specifically of the finger flexors (with digitorum profundus weaker than superficialis) and quadriceps (with vastus medialis and lateralis affected before rectus femoris), but also highlight the pathological characteristics of the disease. The presence of microscopic cellular inflammation (endomysial with invasion of muscle fibres) is the hallmark of the inflammatory nature of IBM. However, the presence of rimmed vacuoles and other 'degenerative' features such as myonuclear degeneration, mitochondrial pathology and myofibrillar cytoplasmic protein aggregates has led to controversy regarding the pathogenesis of the disorder. Over the past several decades, neurologists have often argued in favour of a degenerative origin of IBM (maybe linked to a genetic disorder in common with other vacuolar myopathies with degenerative features, but of unknown molecular origin and not evidenced by recent whole genome sequencing studies) with inflammation being secondary as in certain hereditary myopathies (e.g. dysferlinopathies). Rheumatologists, by contrast, have often favoured an autoimmune origin. In this issue of Brain, Greenberg and co-workers add to evidence supporting the second hypothesis (Greenberg et al., 2019).

The authors elegantly demonstrate that IBM is, from an immunological point of view, clearly different from the other inflammatory myopathies but also (and as expected) from inherited myopathies. They show that IBM is driven by highly differentiated cytotoxic $T$ cells and that this pathogenesis is unique among idiopathic inflammatory myopathies. The work of Greenberg et al. is also important for the methodology used. The authors began by analysing microarray data available from a large series of muscle biopsies from 411 patients: 40 with IBM and 77 with non-IBM idiopathic inflammatory myopathies, 188 with non-inflammatory myopathies including some with hereditary inclusion body myopathy, and 106 with normal muscle. Starting from this genome-wide analysis and using innovative big data analysis methods, Greenberg et al. identified a muscle cytotoxic signature and highly differentiated $T$ cell signature present only in IBM. These cytotoxic and highly differentiated cells were $\mathrm{CD} 8+$ and killer cell lectin-like receptor G1 (KLRG1) positive. The presence of these cells was then verified in the peripheral blood by cytometry analysis and within the muscle by immunohistochemistry. Almost concomitantly, an independent analysis of muscle biopsies from seven patients with IBM and 20 with other inflammatory myopathies confirmed the presence of KLRG1 overexpression in IBM only (Knauss et al., 2019). T-cell receptor (TCR) repertoire analyses have previously shown oligoclonal CD8 $\mathrm{T}$ cell expansion in the peripheral blood and in muscle infiltrates of patients with IBM (Dimitri et al., 2006), while cytometry and immunohistochemistry analyses have shown highly differentiated populations of CD8+ T cells [effector memory (TEM) and terminally differentiated (TEMRA) cells] in blood and muscle. The latter were identified as $\mathrm{CD} 8+\mathrm{CD} 28-$, CD8+CD244+, CD8+CD57+ or CD8+T-bet+ (Allenbach et al., 2014; Dzangué-Tchoupou et al., 2019) and are presumably cytotoxic given their high levels of granzymes and perforin.

The particular strength of the study by Greenberg et al. is the size of its unsupervised genome-wide analysis, 


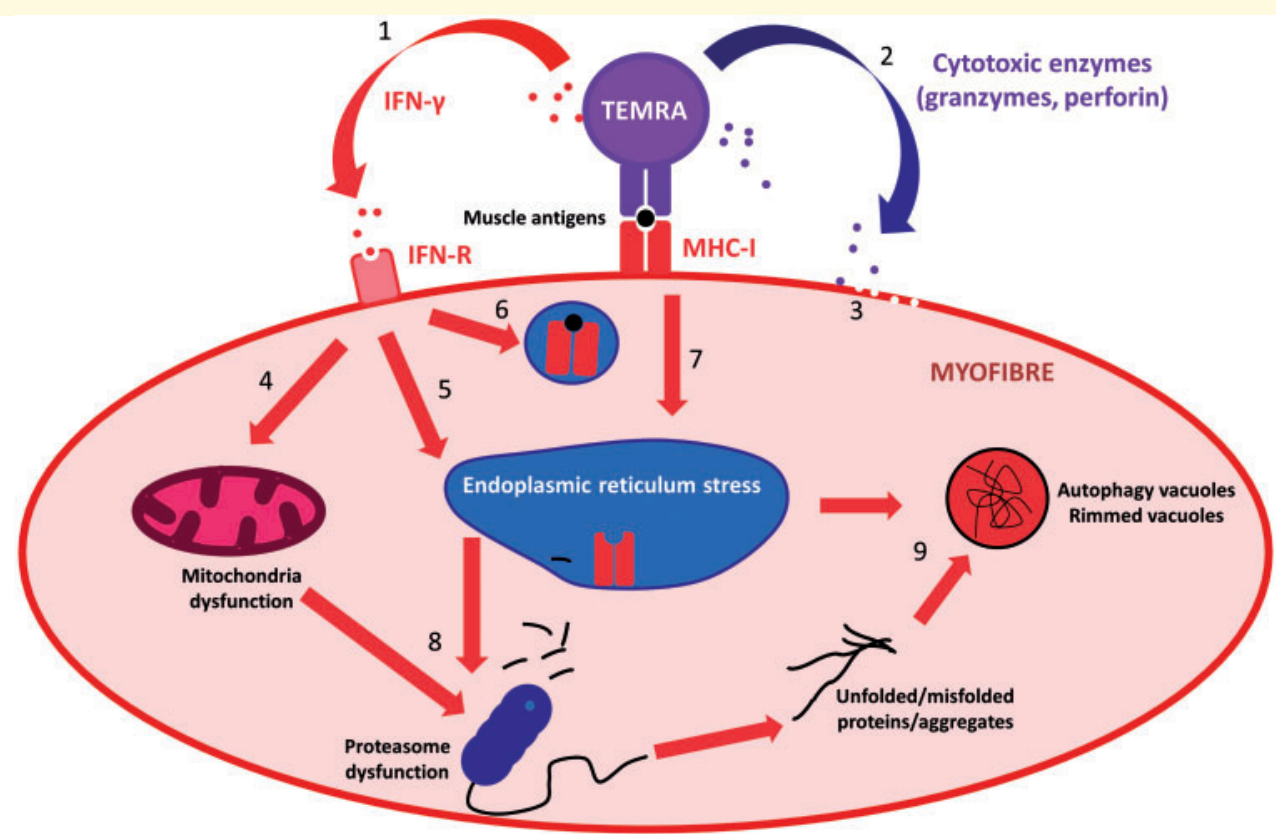

Figure I Putative pathogenesis of IBM. CD8+ terminally differentiated effector memory (TEMRA) T cells are activated by their cognate muscular antigen leading to production of both IFN- $\gamma(\mathrm{I})$ and cytotoxic enzymes e.g. granzymes and perforin (2). Cytotoxic enzymes induce myofibre damage (3). IFN- $\gamma$ may lead to mitochondrial dysfunction (4), endoplasmic reticulum stress (5) and also induces MHC-I overexpression (6). MHC-I overexpression in turn leads to endoplasmic reticulum stress (7). Mitochondrial dysfunction and oxidative stress impair proteasome function (8). Both endoplasmic reticulum stress and proteasome dysfunction contribute to the accumulation of unfolded and misfolded proteins leading to rimmed vacuole formation and degenerative features (9).

which both confirmed the presence of TEMRA cells and also identified a promising cell surface marker of TEM and TEMRA in IBM: KLRG1. It is unfortunate of course that no functional studies were performed on this particular cell population from IBM patients in vitro, ex vivo or in vivo (although IBM has no valid animal model), but the literature and immunostainings in other lymphoid organs are informative. KLRG1 is an inhibitory receptor of the C-type lectin-like family. It is used as a marker of terminally differentiated $\mathrm{NK}$ and $\mathrm{T}$ cells and is strongly induced by chronic viral and other infections. IBM can occur in the setting of viral infection with HIV-1 or human $T$ cell leukaemia virus type 1 (HTLV-1), but in the majority of cases no chronic viral infection is observed. Thus, in IBM, the chronic antigenic stimulation of $\mathrm{T}$ cells must come from some as yet unidentified muscle antigens, presumably presented by the myofibres themselves at their surface by major histocompatibility complex (MHC) class I molecules. MHC class I overexpression is in fact one of the hallmarks of IBM pathology. Thus, in IBM it appears that huge antigen stimulation results over time in transformation of naive or early effector memory $\mathrm{T}$ cells to TEMRA, which can even give rise to the phenotype of $\mathrm{T}$ cell large granular lymphocytic leukaemia. Other autoimmune biomarkers are also observed in IBM, besides the invasion of myofibres by TEMRA KLRG1+ cells. Circulating anti-cN1A autoantibodies have been identified, for example, while genome-wide studies show marked association $\left(P<10^{-33}\right)$ with HLADRB1, an autoimmune haplotype. The highly differentiated CD8+ T cells are resistant to apoptosis, corticosteroids and other immunosuppressants, perhaps explaining why IBM is unresponsive to classical immunosuppressive drugs, similar to Sjögren syndrome and scleroderma, which are without doubt autoimmune diseases.
To close the loop, the degenerative features of IBM should be shown to be secondary to inflammation. And indeed, forced MHC class I overexpression at the surface of myofibres induces endoplasmic reticulum stress and a severe vacuolar myopathy with unfolded protein accumulation (Fréret et al., 2013). Other idiopathic inflammatory myopathies and notably dermatomyositis are also accompanied by mitochondrial dysfunction that is linked to interferon beta (IFN- $\beta$ ) production (Meyer et al., 2017). Another interferon, type II or gamma interferon (IFN- $\gamma$ ) found at high levels within the muscle of IBM by Greenberg et al.'s genome-wide gene expression analysis (Greenberg et al., 2019) and produced by TEMRA cells in IBM (Allenbach et al., 2014), may also induce mitochondrial dysfunction as well as accumulation of amyloid in myofibres (Schmidt et al., 2017). It must be emphasized that IFN- $\gamma$ itself induces MHC class I overexpression at the surface of myofibres. Finally, the 


\section{Glossary}

IBM: Inclusion body myositis is the most prevalent myopathy in individuals over 50 years of age. Its unique phenotype with asymmetrical slowly progressive diffuse muscle weakness mainly affecting the quadriceps, hand finger flexors and swallowing muscles is highly indicative of the diagnosis, and should not be confused with a polymyositis (based on inflammatory infiltrates present on muscle biopsy) because of its resistance to corticosteroids.

KLRG I: Killer cell lectin-like receptor subfamily $G$ member I is a lymphocyte co-inhibitory, or immune checkpoint, receptor expressed predominantly on terminally differentiated effector and effector memory CD8+ T and NK cells.

TEMRA cells: Terminally differentiated effector memory $T$ cells. Human CD8+ $T$ cells are commonly classified into four subsets based on surface expression of the leucocyte common antigen isoform CD45RA and the lymph node addressin CCR7. Naïve T cells (CD45RA+/CCR7+) are thereby distinguished from central memory (TCM, CD45RA-/CCR7+), effector memory (TEM, CD45RA-/CCR7-) and TEMRA (CD45RA+/CCR7-) T cells.

passive transfer of anti-cN1A antibodies in mice or in myofibre cultures induces protein aggregation (Tawara et al., 2017). Figure 1 summarizes the putative pathogenesis of IBM.

The study by Greenberg et al. is also important for having identified KLRG1 cells as potential biomarkers and drug targets for IBM. An increase in the proportion of blood CD8+ $\mathrm{T}$ cells that are also KLRG1+ was found in IBM, just as previous studies have identified increased proportions of blood CD8+ TEMRA cells defined as $\mathrm{CD} 28-$, CD62L-, CD57+, or Tbet+. However, Greenberg et al. do not argue for blood KLRG1+ cells as a diagnostic biomarker of IBM because their presence is likely not specific to this disorder (since the number of TEMRA cells increases with age in the peripheral blood). The measurement of blood KLRG1+ $\mathrm{T}$ cells could potentially serve as a different kind of biomarker; however, such as a clinical trial mechanistic biomarker or surrogate efficacy biomarker, as these cytotoxic cells appear to relate to muscle damage and are easily measured in blood. More importantly, the study does suggest the value of targeting KLRG1+ cells as an IBM therapeutic. In addition to KLRG1 being a marker of presumed pathogenic cells in IBM muscle, the study demonstrated low expression of KLRG1 on lymphoid tissue central memory $\mathrm{T}$ cells, which are of paramount importance for infectious disease defence, and low expression on regulatory $\mathrm{T}$ cells that help to suppress autoimmunity (Greenberg et al., 2019). We can thus imagine that a targeted therapy for IBM that aims to deplete TEMRA KLRG1+ presumably effector cells (e.g. with a monoclonal antibody) may have a good safety profile in terms of infections and may efficiently tackle one of the key actors in IBM pathophysiology.

Olivier Benveniste $e^{1,2,3}$ and

Yves Allenbach ${ }^{1,2,3}$

1 Sorbonne Université, Paris, France

2 INSERM U974, Paris, France

3 Assistance Publique-Hôpitaux de Paris, Paris, France

Correspondence to: Olivier Benveniste E-mail: olivier.benveniste@aphp.fr

doi:10.1093/brain/awz229

\section{Competing interests}

The authors report no competing interests.

\section{References}

Allenbach Y, Chaara W, Rosenzwajg M, Six A, Prevel N, Mingozzi F, et al. Th1 response and systemic treg deficiency in inclusion body myositis. PloS One 2014; 9: e88788.

Dimitri D, Benveniste O, Dubourg O, Maisonobe T, Eymard B, Amoura Z, et al. Shared blood and muscle CD8+ T-cell expansions in inclusion body myositis. Brain 2006; 129: 986-95.

Dzangué-Tchoupou G, Mariampillai K, Bolko L, Amelin D, Mauhin W, Corneau
A, et al. CD8+T-bet+ cells as a predominant biomarker for inclusion body myositis. Autoimmun Rev 2019; 18: 325-33.

Fréret M, Drouot L, Obry A, AhmedLacheheb S, Dauly C, Adriouch S, et al. Overexpression of MHC class I in muscle of lymphocyte-deficient mice causes a severe myopathy with induction of the unfolded protein response. Am J Pathol 2013; 183: 893-904.

Greenberg SA, Pinkus JL, Won Kong S, Baecher-Allan C, Amato AA, Dorfman DM. Highly differentiated cytotoxic T cells in inclusion body myositis. Brain 2019; 142: 2590-604.

Knauss S, Preusse C, Allenbach Y, LeonardLouis S, Touat M, Fischer N, et al. PD1 pathway in immune-mediated myopathies: pathogenesis of dysfunctional $\mathrm{T}$ cells revisited. Neurol Neuroimmunol Neuroinflamm 2019; 6: e558.

Lloyd TE, Mammen AL, Amato AA, Weiss MD, Needham M, Greenberg SA. Evaluation and construction of diagnostic criteria for inclusion body myositis. Neurology 2014; 83: 426-33.

Meyer A, Laverny G, Allenbach Y, Grelet E, Ueberschlag V, Echaniz-Laguna A, et al. IFN- $\beta$-induced reactive oxygen species and mitochondrial damage contribute to muscle impairment and inflammation maintenance in dermatomyositis. Acta Neuropathol 2017; 134: 655-66.

Schmidt K, Wienken M, Keller CW, Balcarek P, Münz C, Schmidt J. IL-1 $\beta$-induced accumulation of amyloid: macroautophagy in skeletal muscle depends on ERK. Mediat Inflamm 2017; 2017: 5470831.

Tawara N, Yamashita S, Zhang X, Korogi M, Zhang Z, Doki T, et al. Pathomechanisms of anti-cytosolic 5'nucleotidase $1 \mathrm{~A}$ autoantibodies in sporadic inclusion body myositis. Ann Neurol 2017; 81: 512-25. 